\title{
Vestibular evoked myogenic potentials testing for diagnosing Sudden Sensorineural Hearing Loss
}

\author{
Madzharova K M.D., Beshkova A. \\ Department of ENT diseases, University hospital Sveti Georgi, \\ Medical University of Plovdiv, Vassil Aprilov Blvd 15 A, 4000 Plovdiv, Bulgaria
}

\begin{abstract}
Sudden sensorineural hearing loss is an emergency condition in otolaryngology. In most cases it manifests as a unilateral reduction in hearing. In addition to hearing loss in one ear, patients often have symptoms such as vertigo and/or tinnitus. The follow-up of patients with sudden sensorineural hearing loss should include not only testing of the hearing system but also diagnosis of the vestibular apparatus. VEMP is an objective method for assessment of the vestibular function through the reaction of the reflex muscle in response to high-intensity acoustic stimulation.

Materials and methods: The subject of study are 12 patients with sudden hearing loss where 5 of the followed patients also complaining of vertigo and instability. After taking a history, the following are performed: examination of ENT organs, pure-tone audiometry, OAE, tympanometry with acoustic reflex, otoneurological examination, VEMP tests, CT (MRI)

Results: Patients with severe sudden hearing loss have deviations in their VEMP tests. The hearing recovery percentage is lower with abnormal VEMP than in those with normal VEMP.

Conclusion: Patients with deviations in VEMP tests can have vestibular disorders in addition to lesions in the organ of Corti, including stria vascularis and the tectorial membrane. There is an association between pathological VEMP and the level of hearing reduction. Abnormal VEMP can be used to predict subsequent incomplete hearing recovery.
\end{abstract}

Keywords: sudden hearing loss, cVEMP, oVEMP, vertigo

\section{Introduction:}

Sudden Sensorineural Hearing Loss (SSHL) is a sound perception reduction of hearing over $30 \mathrm{~dB}$ or more on at least three consecutive frequencies with sudden onset over 3 days, even $\leq 12$ hours $(6,7,8,9,10)$. The etiology can be infections, neoplastic, traumatic, ototoxic, immunological, vascular, psychological $(8,9)$. Vascular causes lead to ischemia and necrosis of the spiral ganglium, degeneration of the organ of Corti. Viral infection in endolymphatic structures, including the cochlea or the spiral ganglion is another possible hypothesis for the etiopathogenesis of this disease. In most cases, it is unilateral for people in their forties without prevalence of either sex. Its incidence in developed countries is estimated at 5-20 cases per 100,000 people/ year. In addition to hearing loss in one ear, patients often have symptoms such as vertigo and/or tinnitus due to anatomic proximity of the two centers $(4,6)$. To improve follow-up of patients with sudden sensorineural hearing loss, attention should not be limited only to examining the cochlea but it is also necessary to include diagnostics and treatment of vestibular abnormalities (6).

VEMP is an additional non-invasive test which examines the function of otolith organs and the vestibulospinal and vestibulo-ocular pathways. cVEMP assesses mainly the saccule and the lower vestibular nerve, while oVEMP assesses the utricle and the upper vestibular nerve (1). VEMP is an objective method for assessment of the vestibular function through the reaction of the reflex muscle 
in response to high-intensity acoustic stimulation. VEMP are registered in all individuals and patients with sensorineural hearing loss who have a normal vestibular apparatus.

The results from studies of patients diagnosed with sudden sensorineural hearing loss indicate that the most commonly damaged vestibular organ is the utricle and the upper vestibular pathway. Patients with deviations in an oVEMP test can have vestibular disorders in addition to lesions in the organ of Corti, including stria vascularis and the tectorial membrane (7). On one hand, Hong et al (2008) find more non-normal VEMP in patients with severe sudden sensorineural hearing loss than with milder hearing loss (3). Stamatiou et al. notes that the severity of the vestibular lesion in cases of sudden sensorineural hearing loss increases with age, a fact associated with degeneration of vestibular structures, which is seen in older individuals (6). He assumes that more severe hearing loss and advanced age act as independent negative prognostic factors for hearing recovery even with or without damage to the labyrinth assessed using cVEMP. In cases of sudden sensorineural hearing loss for vascular reasons, the lesion is expected to be large and to include non only the cochlea but also the vestibular labyrinth damaging a larger number of sensorineural structures (8). Numerous studies describe a positive relationship between a normal VEMP and good prognosis for recovery of hearing in patients with sudden sensorineural hearing loss (8). The percentage of hearing recovery is lower in patients with abnormal VEMP than in those with normal VEMP.

\section{Objective}

Changes in VEMP tests registered in sudden sensorineural hearing loss.

\section{Tasks}

1. To analyze the changes in VEMP tests in case of sudden sensorineural hearing loss.

2. Significance of the study for the diagnostics and prediction of sudden sensorineural hearing loss

\section{Materials and methods}

The subject of study are 12 patients with sudden sensorineural hearing loss, where 5 of the followed patients also complain of vertigo and instability. After taking history, the following are performed: examination of ENT organs, pure-tone audiometry, OAE, tympanometry with acoustic reflex, otoneurological examination, VEMP test, CT (MRI).

\section{Results}

After the examination of ENT organs, Tone Threshold Audiometry is performed. Unilateral sound perception reduction is found in the hearing of all 12 patients and for 6 of them hearing loss is by more than $70 \mathrm{~dB}$ for frequencies from 500 to $8000 \mathrm{~Hz}$. The performed tympanometry indicated a tympanogram type A lacking an acoustic reflex. The conducted otoneurological examination of 5 patients with vertigo found latent vestibular symptoms. No deviations are found in the remaining patients being followed. All patients underwent VEMP testing.

When examining the patients complaining of sudden sensorineural hearing loss in one ear and vertigo, changes are registered in $\mathrm{CVEMP}$ and oVEMP tests. For 5 patients with sudden sensorineural hearing loss without vertigo, deviations are registered - 2 patients show deviations in cVEMP and oVEMP, 2 have atypical oVEMP wave in one ear and 1 has an atypical unilateral wave for cVEMP. Normal VEMP waves are registered for 2 people. Deviations in the oVEMP test are prevalent.

1 month following treatment, control Tone Threshold Audiometry found: 2 patients had socially adequate hearing, 7 had improved with persistent reduced sound perception by the same ear and the hearing of 3 patients had not improved. No deviation is found in the performed otoneurological examination.

Subsequently cVEMP and oVEMP tests are carried out. In 6 of the patients with unilateral sound perception hearing reduction, typical VEMP waves are registered, while 4 of those examined do not complain of vertigo and have no deviations in their otoneurological tests from the first examination. While for 6 people with severe hearing loss, persistent deviations in the VEMP tests are found.

\section{Discussion}

Changes are found when performing VEMP tests of the examined patients with sudden sensorineural hearing loss in one ear who have a vestibular disorder (1). Deviations in the oVEMP test are predominant. Patients with severe sudden sensorineural hearing loss without vertigo are also found to have deviations. Inagaki et al. report that the vestibular 


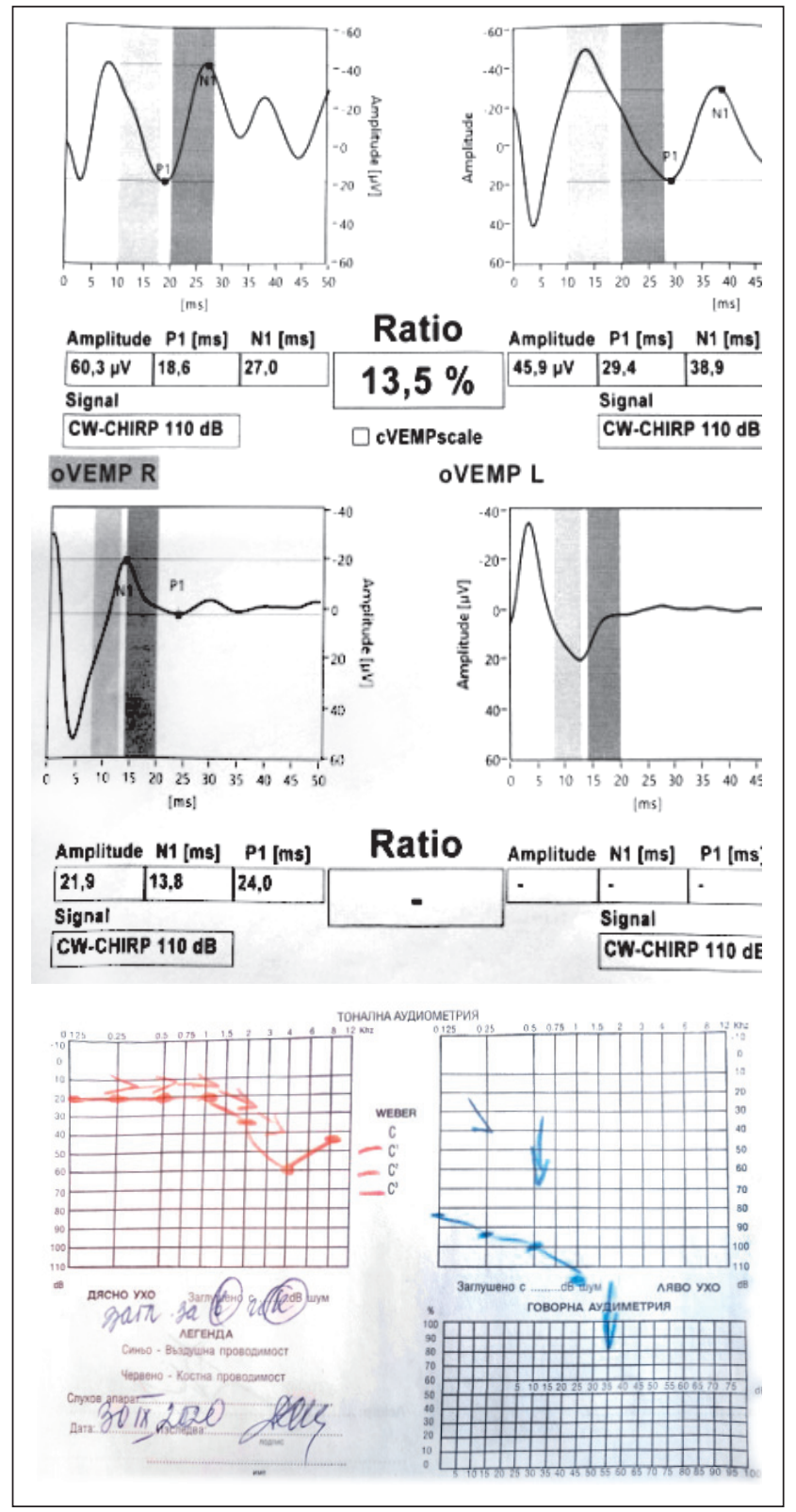

Fig. 1

system is modified in case of sudden sensorineural hearing loss with and without vertigo (4, 7, 8). According to Mohsen Rajati at al. in patients with sudden sensorineural hearing loss without vestibular deviations, pathology is not limited to the cochlea and may also include the sacculus (9). $\mathrm{Wu}, \mathrm{CC}$ and Young, $\mathrm{YH}$ do not find any changes in cVEMP of assessed patients with sudden sensorineural hearing loss (6). Fujimoto et al. (2015) find abnormal oVEMP in $43 \%$ of patients with sudden sensorineural hearing loss. In patients with SSHL, abnormal oVEMP can indicate greater dissemination of the lesion. The association between sudden sensorineural hearing loss and changes in VEMP tests is controversial in scientific literature (8).

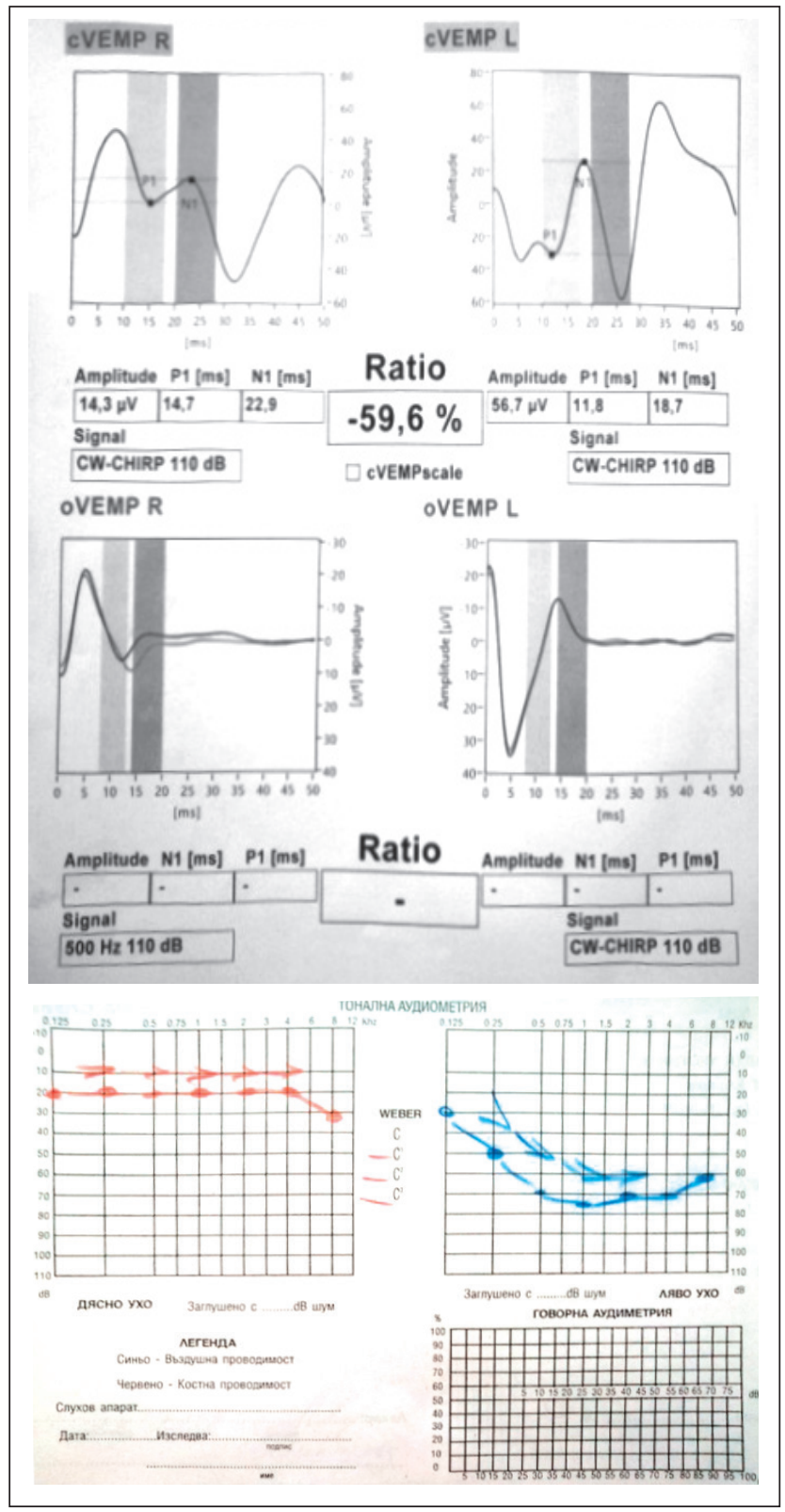

Fig. 2

There is an association between pathological VEMP and the level of hearing reduction. According to Hong et al., for patients with sudden hearing loss over $90 \mathrm{~dB}$ without vestibular deviations, the percentage of registered abnormal VEMP waves is $47 \%$ and for patients with sudden hearing loss and vertigo it is $77 \%$ (4). With greater hearing loss, patients have abnormal results in VEMP tests. In other cases, there is no connection between changes in VEMP and the degree of hearing loss (6). When following-up the examined patients after 1 month post treatment, improvement is seen in the results of the conducted VEMP tests. Normal VEMP waves are registered in patients without vestibular symptoms who have improved results from 
tone threshold audiometry. The study by Noriкo Nagai et al. does not find a significant association between abnormal oVEMP and the degree of hearing loss during the first examination. The rate of hearing recovery is slower in patients with abnormal oVEMP. This result suggests that oVEMP can be used to assess hearing improvement in SSHL (7). Hong et al has also established an association between the altered cVEMP and worse hearing prognosis of patients (6). Iwasaki et al. reports that the lack of cVEMP in 14 of 52 patients with SSHL $(26,9 \%)$ indicates worse hearing recovery $(7)$. The assessment of the vestibular system can be useful in patients with sudden sensorineural hearing loss without vertigo.

\section{Conclusions}

1. Changes are registered in the VEMP tests of patients with severe sudden sensorineural hearing loss in one ear without vestibular deviations

2. Abnormal VEMP can be used to predict subsequent incomplete hearing recovery.

\section{Conclusion}

For the diagnosis and treatment of sudden sensorineural hearing loss, not only testing of the hearing system but also an examination of the vestibular apparatus are significant, regardless of the presence or not of vestibular deviations. The VEMP test can be useful as an additional diagnostic method for patients with sudden sensorineural hearing loss. There is an association between pathological VEMP tests and the level of hearing reduction. The ability of VEMP tests to predict subsequent incomplete hearing recovery is being studied.

Funding: No funding sources

Conflict of interest: None declared

Ethical approval: The study was approved by the Institutional Ethics Committee

\section{Bibliography:}

1. Mario Milkov Applications of vestibular-evoked myogenic potentials in vestibulogy. 1. Basic principles International Bulletin of Otorhinolaryngology 20182 Vol. 14, 34-39

2. Guangwei Zhou MD ScD, Dargie J. BS, Dornan B. AuD and al. Clinical Uses of Cerwical Vestibular-Evoked Myogenic potential Testing in pediatric patient Medicine 2014 July Volume 93 Number 4 PMID: 25068952 PMCID: PMC4602421 DOI: 10.1097/MD.0000000000000037

3. Hain C. Timothy VEMP testing and hearing disorders 2020 July 26, 2020

4. Hong Seok Min, MD, Byun Jae Yong MD, PhD, Chan Hum Park, MD, PhD, Jun Lee,MD, Park Moon Suh, MD, PhD and Chang II Cha MD, $\mathrm{PhD}$, Saccular Damage in patients with idiopathic sudden sensorineural hearing loss without vertigo, Otolaryngology-Head and Neck Surgery $2008139,541-545$

5. Ledesma Alleluia Lima Losno, Barreto Monique Antunes de Souza Chelminski, Fayez Bahmad, Jr V estibular evoked myogenic potential: its use in Sudden Sensorineural Hearing Loss International Tinnitus Journal. 2014; 19 (1):82-85

6. Maia Natalia de Paula Doyle, Lopes Karen de Carvalho, Fernando Freitas Gananca, Vestibular evoked myogenic potentials in the prognosis of sudden hearing loss-a systematic review Brazilian Journal of Otorhinolaryngology 2020; 86(2) 247-254

7. NagaI Noriko, Ogawa Yasuo, Akira Hagiwara, Koji Otsuka, Taro Inagaki, Shigetaka Shimizu and Mamoru Susuki Ocular vestibular evoked myogenic potentials induced by bone-conducted vibration in patients with unilateral inner ear disease Acta Oto-Laryngologica. 2014; 134: $151-158$

8. Oiticica Jeanne, Bittar Roseli Saraiva Moreira, Claudio Campi de Castro, Signe Grasel, Larissa Vilela Pereira, Sandra Lira Bastos, Alice Carolina Mataruco Ramos, Roberto Beck. Contribution of audiovestibular tests to the topographic diagnosis of sudden deafness Int. Arch. Otorhinolaryngol. 2013;17(3):305-314.

9. Rajati Mohsen, Mehdi Bakhshaee, Ebrahim Naghavi, Faezeh Hoseinnejad, Hamid Reza Rouhi, Rahman Movahhed. Studying VEMP in Sudden Sensorineural Hearing Loss Iranian Journal of Otorhinolaryngology 2014 No.3, Vol.23, Serial No.64

10. Wang Yuan, Gu Shun-Tong, Bao Xiao-Lin and Jia-Liang Guo. Clinical value of vestibular-evoked myogenic potential tests in patients with sudden sensorineural hearing loss BMC Neurol. 201919337 doi: 10 1186/s12883-019-1576-Z

11. Zhou Yujuan, Wu Yongzhen, Wang Zing. Otolitic organ function in patients with profound sensorineural hearing loss Journal of otology 11 2016 73-77 